\title{
Characterization of a novel rat cytomegalovirus (RCMV) infecting placenta-uterus of Rattus rattus diardii
}

\begin{abstract}
A new rat cytomegalovirus (RCMV) isolated from the placenta/uterus of a house rat (Rattus rattus diardii) was found to productively infect rat embryo fibroblast (REF) cells. The virus produced typical herpesvirus-like cytopathic effects characterized by a lytic infection. The well-known herpesvirus morphology was confirmed by electron microscopy. Its slow growth in cell culture indicated that the virus is belonging to subfamily Betaherpesvirinae. Electron microscopy techniques and immunohistochemistry confirmed the presence of herpesviral inclusion bodies and virus related particles in the cytoplasm and nucleus of infected cells. Hyperimmune serum against the Maastricht strain of RCMV revealed the virus identity in neutralization test, immunoperoxidase and immunofluorescence techniques. Despite typical characteristics of CMV, the viral genome is significantly different from that of Maastricht, English, UPM/Sg and UPM/Kn strains. The dissimilarities, which have not been reported before, had been confirmed by mean of restriction endonuclease analysis. The new RCMV strain, a virus that infects placenta and uterus of rats, has been named as ALL-03.
\end{abstract}

